International Journal of Pure and Applied Mathematics

Volume 112 No. 3 2017, 469-480

ISSN: 1311-8080 (printed version); ISSN: 1314-3395 (on-line version)

url: http://www.ijpam.eu

doi: 10.12732 /ijpam.v112i3.2

\title{
EXISTENCE OF WEAK SOLUTIONS FOR
}

\section{A FUNCTIONAL INTEGRAL INCLUSION}

\author{
Ahmed M.A. El-Sayed ${ }^{1}$, Nesreen F.M. El-Haddad ${ }^{2} \S$ \\ ${ }^{1}$ Faculty of Science \\ Alexandria University \\ Alexandria, EGYPT \\ ${ }^{2}$ Faculty of Science \\ Damanhour University \\ Behera, EGYPT
}

Abstract: In this paper we study the existence of weak solution $x \in C[I, E]$ for the nonlinear functional integral inclusion

$$
x(t) \in F\left(t, \int_{0}^{t} g(s, x(m(s))) d s\right), \quad t \in[0, T]
$$

in the reflexive Banach space $E$ under the assumption that the set-valued function $F$ satisfy Lipschitz condition.

AMS Subject Classification: 45N05, 46T20, 47J05

Key Words: set-valued function, weak solution, functional inclusion, Lipschitz condition

\section{Introduction}

Let $I=[0, T]$, and let $L^{1}(I)$ be the class of all Lebesgue integrable functions defined on the interval $I$. Let $E$ be a reflexive Banach space with norm $\|$.$\| and$ dual $E^{*}$.

Denote $C[I, E]$ the Banach space of strongly continuous functions $x: I \rightarrow E$ with sup-norm $\|x\|_{C}=\sup \|x(t)\|_{E}$.

Received: $\quad$ August 28, 2016

Revised: December 1, 2016

Published: $\quad$ February 7, 2017

$\S_{\text {Correspondence author }}$ (c) 2017 Academic Publications, Ltd. url: www.acadpubl.eu 
Consider the functional integral inclusion

$$
x(t) \in F\left(t, \int_{0}^{t} g(s, x(m(s))) d s\right), \quad t \in[0, T],
$$

where $F: I \times E \rightarrow P(E)$ is a nonlinear set-valued mapping, and $P(E)$ denote the family of nonempty subsets of the Banach space $E$.

Here we study the existence of weak solution $x \in C[I, E]$ of the functional integral inclusion (1) in the reflexive Banach space $E$, under the assumption that the set-valued function $F$ satisfy Lipschitz condition.

Indeed a set-valued functional equations have been extensively investigated by a number of authors and there are many interesting results concerning this problem (see [3], [8]-[10] and [12]-[13]).

The existence of weak solutions of the integral equations were studied by a number of authors such as (see for instance[1]-[2], [5] and [15]-[16]).

\section{Preliminaries}

Here, we present some auxiliary results that will be needed in this work.

Let $E$ be a Banach space and let $x: I \rightarrow E$, then:

(1) $x($.$) is said to be weakly continuous (measurable) at t_{0} \in I$ if for every $\phi \in E^{*}, \phi(x()$.$) is continuous (measurable) at t_{0}$.

(2) A function $h: E \rightarrow E$ is said to be weakly sequentially continuous if $h$ maps weakly convergent sequence in $E$ to weakly convergent sequence in $E$.

If $x$ is weakly continuous on $I$, then $x$ is strongly measurable and hence weakly measurable (see[4] and[7]). Note that in reflexive Banach spaces weakly measurable functions are pettis integrable (see[7] and [11] for the definition) if and only if $\phi(x()$.$) is Lebesgue integrable on I$ for every $\phi \in E^{*}$.

Now we state a fixed point theorem and some propositions which will be used in the sequal (see[14]).

Theorem 1 (O'Regan Fixed Point Theorem). Let $E$ be a Banach space and let $Q$ be a nonempty, bounded, closed and convex subset of the space $(C[0, T], E)$ and let $A: Q \rightarrow Q$ be a weakly sequentially continuous and assume that $A Q(t)$ is relatively weakly compact in $E$ for each $t \in[0, T]$. Then $A$ has a fixed point in the set $Q$.

Proposition 2. A subset of a reflexive Banach space is weakly compact if and only if it is closed in the weak topology and bounded in the norm topology. 
Proposition 3. Let $E$ be a normed space with $y \neq 0$. Then there exists a $\phi \in E^{*}$ with $\|\phi\|=1$ and $\|y\|=\phi(y)$.

Definition 4 (see [6]). A set-valued map $F$ from $I \times E$ to the family of all nonempty closed subsets of $E$ is called Lipschitzian if there exists $L>0$ such that for all $t_{1}, t_{2} \in I$ and all $x_{1}, x_{2} \in E$, we have

$$
H\left(F\left(t_{1}, x_{1}\right), F\left(t_{2}, x_{2}\right)\right) \leq L\left(\left|t_{1}-t_{2}\right|+\left\|x_{1}-x_{2}\right\|\right)
$$

where $H(A, B)$ is the Hausdorff metric between the two subsets $A, B \in I \times E$.

Denote $S_{F}=\operatorname{Lip}(I, E)$ be the set of all Lipschitz selections of $F$.

\section{Existence of Weak Solution}

In this section, we present our main result by proving the existence of weak solution $x \in C[I, E]$ of the functional integral inclusion (1) in the reflexive Banach space $E$, under the assumption that the set-valued function $F$ satisfy Lipschitz condition.

\subsection{Coupled System Approach}

Consider now the functional integral inclusion (1) under the following assumptions:

(H1) The set $F(t, x)$ is compact and convex for all $(t, x) \in I \times E$.

(H2) The set-valued map $F$ is Lipschitzian with a Lipschitz constant $L>0$.

(H3) The set of all Lipschitz selections $S_{F}$ is nonempty.

(H4) $g(t,$.$) is weakly sequentially continuous for each t \in I$.

(H5) $g(., x)$ is weakly measurable on $I$ for every $x \in E$.

(H6) There exists a function $a \in L^{1}[0, T]$ and a constant $b>0$ such that

$$
\|g(t, x)\| \leq|a(t)|+b\|x\|, \quad \forall t \in I
$$

(H7) $m:[0, T] \rightarrow[0, T]$ is continuous.

Remark 5. From assumptions (H2) and (H3), there exists $f \in S_{F}$ such that

$$
\left\|f\left(t_{2}, x\right)-f\left(t_{1}, y\right)\right\| \leq L\left(\left|t_{2}-t_{1}\right|+\|x-y\|\right)
$$


and

$$
x(t)=f\left(t, \int_{0}^{t} g(s, x(m(s))) d s, \quad t \in[0, T] .\right.
$$

Then the solution of the functional integral equation (3), if it exists, is a solution of the functional integral inclusion (1).

Now let

$$
y(t)=\int_{0}^{t} g(s, x(m(s))) d s, \quad t \in[0, T],
$$

then from (3) we have

$$
x(t)=f(t, y(t)), \quad t \in[0, T] .
$$

Then the functional integral equation (3) is equivalent to the coupled system (4) and (5).

Consider now the coupled system (4) and (5).

Now, we study the existence of a weak solution of the functional integral equation (3), which is a solution of the functional integral inclusion (1), by getting the weak solution of the coupled system (4) and (5).

Definition 6. By a weak solution of the coupled system (4) and (5) we mean the ordered pair of functions $(x, y), x, y \in C[I, E]$ such that

$$
\begin{gathered}
\phi(x(t))=\phi(f(t, y(t))), \quad t \in[0, T] \\
\phi(y(t))=\int_{0}^{t} \phi(g(s, x(m(s)))) d s, \quad t \in[0, T]
\end{gathered}
$$

for all $\phi \in E^{*}$.

Now let $X$ be the class of all ordered pair $U=(x, y), x, y \in C[I, E]$, with norm

$$
\|(x, y)\|_{X}=\|x\|+\|y\|, \quad x, y \in C[I, E] .
$$

Now for the existence of a weak continuous solution of the coupled system (4) and (5) we have the following theorem.

Theorem 7. Let the assumptions (H1)-(H7) be satisfied. Then the coupled system (4) and (5) has at least one weak continuous solution. 
Proof. Let

$$
\begin{aligned}
U(t) & =(x(t), y(t)) \\
& =\left(f(t, y(t)), \quad \int_{0}^{t} g(s, x(m(s))) d s\right), \quad t \in[0, T]
\end{aligned}
$$

Let $A$ be any operator defined by

$$
A U(t)=A(x(t), y(t))=\left(A_{1} y(t), A_{2} x(t)\right)
$$

where

$$
\begin{gathered}
A_{1} y(t)=f(t, y(t)), \quad t \in[0, T] \\
A_{2} x(t)=\int_{0}^{t} g(s, x(m(s))) d s, \quad t \in[0, T]
\end{gathered}
$$

Let the set $Q_{r}$ defined by

$$
Q_{r}=\left\{U=(x, y) \in X: x, y \in C[I, E], \quad\|y\| \leq r_{1},\|x\| \leq r_{2}, \quad r=r_{1}+r_{2}\right\} .
$$

Let $U=(x, y) \in Q_{r}$ be an arbitrary ordered pair, then we have from proposition 3

$$
\begin{aligned}
\left\|A_{1} y(t)\right\| & =\phi\left(A_{1} y(t)\right) \\
& =\phi(f(t, y(t))) \\
& =\|f(t, y(s))\| \\
& \leq L\|y\|+\sup |f(t, 0)| \\
& \leq L\|y\|+M_{1},
\end{aligned}
$$

where $M_{1}=\sup |f(t, 0)|$. Then

$$
\left\|A_{1} y(t)\right\| \leq L r_{1}+M_{1}=r_{1}, \text { where } r_{1}=\frac{M_{1}}{1-L} .
$$

And

$$
\begin{aligned}
\left\|A_{2} x(t)\right\| & =\phi\left(A_{2} x(t)\right) \\
& =\int_{0}^{t} \phi(g(s, x(m(s)))) d s \\
& =\int_{0}^{t}\|g(s, x(m(s)))\| d s \\
& \leq \int_{0}^{t}\{|a(s)|+b\|x(m(s))\|\} d s
\end{aligned}
$$




$$
\begin{aligned}
& \leq \int_{0}^{t}|a(s)| d s+b \int_{0}^{t}\|x(m(s))\| d s \\
& \leq M_{2}+b r_{2} T .
\end{aligned}
$$

Therefore

$$
\left\|A_{2} x(t)\right\| \leq M_{2}+b r_{2} T=r_{2}, \text { where } r_{2}=\frac{M_{2}}{1-b T}
$$

Now

$$
\begin{aligned}
\|A U(t)\|_{X} & =\left\|A_{1} y(t)\right\|+\left\|A_{2} x(t)\right\| \\
& \leq M_{1} T+L r_{1}+M_{2}+b r_{2} T \\
& =r .
\end{aligned}
$$

Then

$$
\|A U\|_{X} \leq r
$$

Hence, $A U \in Q_{r}$, which proves that $A Q_{r} \subset Q_{r}$, i.e. $A: Q_{r} \rightarrow Q_{r}$, and the class of functions $\left\{A Q_{r}\right\}$ is uniformly bounded.

Now $Q_{r}$ is nonempty, closed, convex and uniformly bounded.

As a consequence of proposition 2 , then $\left\{A Q_{r}\right\}$ is relatively weakly compact.

Now, we shall prove that $A: X \rightarrow X$.

Let $t_{1}, t_{2} \in I, \quad t_{1}<t_{2}$ (without loss of generality assume that $\| A U\left(t_{2}\right)-$ $\left.A U\left(t_{1}\right) \| \neq 0\right)$, then firstly we have

$$
\begin{aligned}
\left\|A_{1} y\left(t_{2}\right)-A_{1} y\left(t_{1}\right)\right\| & =\left\|f\left(t_{2}, y\left(t_{2}\right)\right)-f\left(t_{1}, y\left(t_{1}\right)\right)\right\| \\
& \leq L\left(\left|t_{2}-t_{1}\right|+\left\|y\left(t_{2}\right)-y\left(t_{1}\right)\right\|\right)
\end{aligned}
$$

and

$$
\begin{aligned}
\left\|A_{2} x\left(t_{2}\right)-A_{2} x\left(t_{1}\right)\right\| & =\phi\left(A_{2} x\left(t_{2}\right)-A_{2} x\left(t_{1}\right)\right) \\
& =\int_{t_{1}}^{t_{2}} \phi(g(s, x(m(s)))) d s \\
& =\int_{t_{1}}^{t_{2}}\|(g(s, x(m(s))))\| d s \\
& \leq \int_{t_{1}}^{t_{2}}\{|a(s)|+b\|x(m(s))\|\} d s \\
& \left.\leq \int_{t_{1}}^{t_{2}}|a(s)| d s+b \int_{t_{1}}^{t_{2}}\|x(m(s))\|\right\} d s
\end{aligned}
$$




$$
\leq \int_{t_{1}}^{t_{2}}|a(s)| d s+b r_{2}\left|t_{2}-t_{1}\right| .
$$

Then

$$
\begin{aligned}
\left\|A U\left(t_{2}\right)-A U\left(t_{1}\right)\right\|_{X} & =\left\|\left(A_{1} y\left(t_{2}\right), A_{2} x\left(t_{2}\right)\right)-\left(A_{1} y\left(t_{1}\right), A_{2} x\left(t_{1}\right)\right)\right\| \\
& =\left\|\left(\left(A_{1} y\left(t_{2}\right)-A_{1} y\left(t_{1}\right)\right),\left(A_{2} x\left(t_{2}\right)-A_{2} x\left(t_{1}\right)\right)\right)\right\| \\
& =\left\|A_{1} y\left(t_{2}\right)-A_{1} y\left(t_{1}\right)\right\|+\left\|A_{2} x\left(t_{2}\right)-A_{2} x\left(t_{1}\right)\right\| .
\end{aligned}
$$

Which proves that $A: X \rightarrow X$.

Finally, we want to prove that $A$ is weakly sequentially continuous.

Let $\left\{U_{n}\right\}$ be a sequence in $Q_{r}$ converges weakly to $U \forall t \in I$, then we have the two sequences $\left\{y_{n}\right\},\left\{x_{n}\right\}$, such that $\left\{y_{n}\right\}$ converges strongly to $y$ and $\left\{x_{n}\right\}$ converges weakly to $x$, i.e. $y_{n}(t) \rightarrow y, x_{n}(t) \rightarrow x, \quad \forall t \in I$.

Since $g(t, x(m(t)))$ is weakly sequentially continuous in $x$, then $g\left(t, x_{n}(m(t))\right)$ converges weakly to $g(t, x(m(t)))$.

Thus $\phi\left(g\left(t, x_{n}(m(t))\right)\right)$ converges strongly to $\phi(g(t, x(m(t))))$, and $\phi\left(f\left(t, y_{n}(t)\right)\right)$ converges strongly to $\phi(f(t, y(t)))$.

Also,

$$
\left\|g\left(t, x_{n}(m(s))\right)\right\| \leq|a(t)|+b\left\|x_{n}\right\|
$$

By applying Lebesgue dominated convergence theorem for Pettis integral, then we get

$$
\begin{aligned}
\phi\left(A_{1} y_{n}(t)\right) & =\phi\left(f\left(t, y_{n}(t)\right)\right) \\
& =\left\|f\left(t, y_{n}(t)\right)\right\| \\
& \rightarrow\|f(t, y(t))\|, \quad \forall \phi \in E^{*}, \quad t \in I
\end{aligned}
$$

i.e. $\phi\left(A_{1} y_{n}(t)\right) \rightarrow \phi\left(A_{1} y(t)\right)$, and then

$$
\left\|A_{1} y_{n}(t)\right\| \rightarrow\left\|A_{1} y(t)\right\|
$$

and

$$
\begin{aligned}
\phi\left(\int_{0}^{t} g\left(s, x_{n}(m(s))\right) d s\right) & =\int_{0}^{t} \phi\left(g\left(s, x_{n}(m(s))\right)\right) d s \\
& =\int_{0}^{t}\left\|g\left(s, x_{n}(m(s))\right)\right\| d s \\
& \rightarrow \int_{0}^{t}\|g(s, x(m(s)))\| d s, \quad \forall \phi \in E^{*}, \quad t \in I
\end{aligned}
$$


i.e. $\phi\left(A_{2} x_{n}(t)\right) \rightarrow \phi\left(A_{2} x(t)\right)$, and then

$$
\left\|A_{2} x_{n}(t)\right\| \rightarrow\left\|A_{2} x(t)\right\| .
$$

Therefore,

$$
\begin{aligned}
\left\|A U_{n}(t)\right\|_{X} & =\left\|A\left(x_{n}(t), y_{n}(t)\right)\right\| \\
& =\left\|\left(A_{1} y_{n}(t), A_{2} x_{n}(t)\right)\right\| \\
& =\left\|A_{1} y_{n}(t)\right\|+\left\|A_{2} x_{n}(t)\right\| \\
& \rightarrow\left\|A_{1} y(t)\right\|+\left\|A_{2} x(t)\right\| \\
& \rightarrow\left\|\left(A_{1} y(t), A_{2} x(t)\right)\right\| \\
& \rightarrow\|A U(t)\|_{X}
\end{aligned}
$$

Hence, $A$ is weakly sequentially continuous (i.e. $A U_{n}(t) \rightarrow A U(t), \quad \forall t \in I$ weakly).

Since all conditions of O'Regan theorem are satisfied, then the operator $A$ has at least one fixed point $U \in Q_{r}$, and then the coupled system (4) and (5) has at least one weak solution $(x, y) \in X$, then there exists at least one weak solution $x \in C[I, E]$ of the functional integral equation (3).

Consequently, there exists at least one weak continuous solution $x \in C[I, E]$ of the functional integral inclusion (1).

\subsection{Functional Integral Inclusion Approach}

Definition 8. By a solution of the functional integral inclusion (1) we mean the function $x \in C[I, E]$ satisfying (1).

Now for the existence of weak solution $x \in C[I, E]$ of the functional integral inclusion (1) we have the following theorem.

Theorem 9. Let the assumptions (H1)-(H7) be satisfied, then there exists at least one weak solution $x \in C[I, E]$ of the functional integral inclusion (1).

Proof. Let the set-valued function $F$ satisfy the assumptions (H1)-(H3), then there exists $f \in S_{F}$ such that

$$
\left\|f\left(t_{2}, x\right)-f\left(t_{1}, y\right)\right\| \leq L\left(\left|t_{2}-t_{1}\right|+\|x-y\|\right),
$$

And $f$ satisfy the functional integral equation (3)

Define the operator $A$ by

$$
A x(t)=f\left(t, \int_{0}^{t} g(s, x(m(s))) d s\right), \quad t \in I
$$


Let the set $Q_{r}$ defined by

$$
Q_{r}=\{x \in C[I, E], \quad\|x\| \leq r\} .
$$

Let $x \in Q_{r}$ be arbitrary, then we have from proposition 3

$$
\begin{aligned}
\|A x(t)\| & =\phi(A x(t)) \\
& =\phi f\left(t, \int_{0}^{t} g(s, x(m(s))) d s\right) \\
& =\left\|f\left(t, \int_{0}^{t} g(s, x(m(s))) d s\right)\right\| \\
& \leq L\left\|\int_{0}^{t} g(s, x(m(s))) d s\right\|+\sup |f(t, 0)| \\
& \leq L \int_{0}^{t}\|g(s, x(m(s)))\| d s+\sup |f(t, 0)| \\
& \leq L \int_{0}^{t}\{|a(s)|+b\|x\|\} d s+\sup |f(t, 0)| \\
& \leq L\left\{\int_{0}^{t}|a(s)| d s+b \int_{0}^{t}\|x\| d s\right\}+\sup |f(t, 0)| \\
& \leq L\left\{M_{2}+b \operatorname{Tr}\right\}+M_{1}
\end{aligned}
$$

where $M_{1}=\sup |f(t, 0)|, M_{2}=\int_{0}^{t}|a(t)| d s$.

Therefore

$$
\|A x(t)\| \leq L M_{2}+L b T r+M_{1}=r, \text { where } r=\frac{L M_{2}+M_{1}}{1-L b T} .
$$

Then

$$
\|A x(t)\| \leq r .
$$

Hence, $A x \in Q_{r}$, which proves that $A Q_{r} \subset Q_{r}$, i.e. $A: Q_{r} \rightarrow Q_{r}$, and the class of functions $\left\{A Q_{r}\right\}$ is uniformly bounded.

Now $Q_{r}$ is nonempty, closed, convex and uniformly bounded.

As a consequence of proposition 1 , then $\left\{A Q_{r}\right\}$ is relatively weakly compact.

Now, we shall prove that $A: C[I, E] \rightarrow C[I, E]$.

Let $t_{1}, t_{2} \in I, \quad t_{1}<t_{2}$ (without loss of generality assume that $\| A x\left(t_{2}\right)-$ $\left.A x\left(t_{1}\right) \| \neq 0\right)$, then we have

$$
\left\|A x\left(t_{2}\right)-A x\left(t_{1}\right)\right\|=\left\|f\left(t_{2}, \int_{0}^{t_{2}} g(s, x(m(s))) d s\right)-f\left(t_{1}, \int_{0}^{t_{1}} g(s, x(m(s))) d s\right)\right\|
$$




$$
\begin{aligned}
& \leq L\left(\left|t_{2}-t_{1}\right|+\left\|\int_{0}^{t_{2}} g(s, x(m(s))) d s-\int_{0}^{t_{1}} g(s, x(m(s))) d s\right\|\right) \\
& \leq L\left(\left|t_{2}-t_{1}\right|+\left\|\int_{t_{1}}^{t_{2}} g(s, x(m(s))) d s\right\|\right) \\
& \leq L\left(\left|t_{2}-t_{1}\right|+\int_{t_{1}}^{t_{2}}\|g(s, x(m(s)))\| d s\right) \\
& \leq L\left(\left|t_{2}-t_{1}\right|+\int_{t_{1}}^{t_{2}}\{|a(s)|+b\|x\|\} d s\right) \\
& \leq L\left(\left|t_{2}-t_{1}\right|+\int_{t_{1}}^{t_{2}}|a(s)| d s+b \int_{t_{1}}^{t_{2}}\|x\| d s\right) \\
& \leq L\left(\left|t_{2}-t_{1}\right|+\int_{t_{1}}^{t_{2}}|a(s)| d s+b r\left|t_{2}-t_{1}\right|\right) .
\end{aligned}
$$

Then

$$
\left\|A x\left(t_{2}\right)-A x\left(t_{1}\right)\right\| \leq L\left(\left|t_{2}-t_{1}\right|+\int_{t_{1}}^{t_{2}}|a(t)| d s+b r\left|t_{2}-t_{1}\right|\right) .
$$

Which proves that $A: C[I, E] \rightarrow C[I, E]$.

Finally, we want to prove that $A$ is weakly sequentially continuous.

Let $\left\{x_{n}\right\}$ be a sequence in $Q_{r}$ converges weakly to $x \quad \forall t \in I$, i.e. $x_{n}(t) \rightarrow$ $x, \quad \forall t \in I$.

Since $g(t, x(m(t)))$ is weakly sequentially continuous in $x$, then $g\left(t, x_{n}(m(t))\right)$ converges weakly to $g(t, x(m(t)))$.

Thus $\phi\left(g\left(t, x_{n}(m(t))\right)\right)$ converges strongly to $\phi(g(t, x(m(t))))$.

Also,

$$
\left\|g\left(t, x_{n}(m(s))\right)\right\| \leq|a(t)|+b\left\|x_{n}\right\|
$$

Then by applying Lebesgue dominated convergence theorem for Pettis integral, we get

$$
\begin{aligned}
\phi\left(A x_{n}(t)\right) & =\phi\left(f\left(t, \int_{0}^{t} g\left(s, x_{n}(m(s))\right) d s\right)\right) \\
& =\left\|f\left(t, \int_{0}^{t} g\left(s, x_{n}(m(s))\right) d s\right)\right\| \\
& \rightarrow\left\|f\left(t, \int_{0}^{t} g(s, x(m(s))) d s\right)\right\|, \quad \forall \phi \in E^{*}, \quad t \in I
\end{aligned}
$$

i.e. $\phi\left(A x_{n}(t)\right) \rightarrow \phi(A x(t))$, and then

$$
\left\|A x_{n}(t)\right\| \rightarrow\|A x(t)\|
$$


Hence, $A$ is weakly sequentially continuous (i.e. $A x_{n}(t) \rightarrow A x(t), \quad \forall t \in I$ weakly).

Since all conditions of O'Regan theorem are satisfied, then the operator $A$ has at least one fixed point $x \in Q_{r}$, and then there exists at least one weak solution $x \in C[I, E]$ of the functional integral equation (3).

Consequently, there exists at least one weak solution $x \in C[I, E]$ of the functional integral inclusion (1).

\section{References}

[1] D. Bugajewski, On the existence of weak solutions of integrl equations in Banach spaces, Comment. Math. Univ. Carolin, 35 (1994),35-41.

[2] M. Cichon, I. Kubiaczyk, Existence theorem for the Hammerstien integral equation, Discuss. Math. Diff. Incl. Control and optimization, 16(1996), 171-177.

[3] T.Cardinali, K. Nikodem and F. Papalini, Some results on stability and characterization of k-convexity of set-valued functions, Ann. Polon, Math. 58 (1993), 185-192.

[4] N. Dunford, J. T. Schwartz, Linear operators, Interscience, Wiley, New York, (1958).

[5] A. M. A. EL-Sayed, H. H. G. Hashem, Weak maximal and minimal solutions for Hammerstien and Urysohn integral equations in reflexive Banach spaces, In: Differential equation and control processes, 4(2008), 50-62.

[6] A. M. A. El-sayed and A. G. Ibrahim, Multivalued fractional differential equations, In: Applied Mathematics and Computation, 68 (1995), 15-25.

[7] E. Hille, R. S. Phillips, Functional Analysis and Semi-groups, Amer. Math. Soc. Colloq. Publ. 31,Amer. Math. Soc., Providence, R. I. (1957).

[8] K.Nikodem, On quadratic set-valued functions, Publ. Math. Debrecen, 30 (1984), 297301.

[9] K. Nikodem, On jensen's functional equation for set-valued functions, Rad.Math, $\mathbf{3}$ (1987), 23-33.

[10] K. Nikodem, Set-valued solutions of the pexider functional equations, Funkcial.Ekvac, 31 (1988), 227-231.

[11] B. J. Pettis, On integration in vector spacecs, Trans. Amer. Math. Soc., 44 (1938), 277-304.

[12] D.Popa, Functional inclusions on square symmetric grupoids and Hyers-Ulam stability, Mathematical Inequal \&3 Appl., 7 (2004), 419-428.

[13] D.Popa, Aproperty of a functional inclusion connected with Hyers-Ulam stability. J. Math. Inequal, 4 (2009), 591-598.

[14] D. O'Regan, Fixed point theory for wealy sequentially continuous mapping, Math. Comput. Modeling, 27 (1998), 1-14. 
[15] D. O'Regan, Integral equations in reflexive Banach spaces and weak topologies, Amer. Mah. Soc., 2 (1996), 607-614.

[16] H.A.H. Salem, A. M. A. EL-Sayed, Fractional order integral equations in reflexive Banach spaces, Math. Slovaca., 2 (2005), 169-181. 